\title{
Parameterized Synthesis Case Study: AMBA AHB
}

\author{
Roderick Bloem Swen Jacobs Ayrat Khalimov \\ Graz University of Technology, Austria * \\ firstname. lastname@iaik.tugraz.at
}

\begin{abstract}
We revisit the AMBA AHB case study that has been used as a benchmark for several reactive synthesis tools. Synthesizing AMBA AHB implementations that can serve a large number of masters is still a difficult problem. We demonstrate how to use parameterized synthesis in token rings to obtain an implementation for a component that serves a single master, and can be arranged in a ring of arbitrarily many components. We describe new tricks - property decompositional synthesis, and direct encoding of simple GR(1) - that together with previously described optimizations allowed us to synthesize a component model with 14 states in about 1 hour.
\end{abstract}

\section{Introduction}

By automatically generating correct implementations from a temporal logic specification, reactive synthesis tools can relieve system designers from tedious and error-prone tasks like low-level manual implementation and debugging. This great benefit comes at the cost of high computational complexity of synthesis, which makes synthesis of large systems an ambitious goal. For instance, Bloem et al. [6] synthesize an arbiter for the ARM AMBA Advanced High Performance Bus (AHB) [2]. The results, obtained using RATSY [4], show that both the size of the implementation and the time for synthesis increase steeply with the number of masters that the arbiter can handle. This is unexpected, since an arbiter for $n+1$ masters is very similar to an arbiter for $n$ masters, and manual implementations grow only slightly with the number of masters. While recent results show that synthesis time and implementation size can be improved in standard LTL synthesis tools [8, 10], the fundamental problem of increasing complexity with the number of masters can only be solved by adapting the synthesis approach itself.

To this end, Jacobs and Bloem [11] introduced the parameterized synthesis approach. In parameterized synthesis, we synthesize a component implementation that can be used as a building block, replicating components to form a system that satisfies a specification for any number of components. The approach is based on cutoff results that have previously only been used to reduce the verification of parameterized systems to systems of a small, fixed size. In particular, small cutoffs exist for token-ring networks, as shown by Emerson and Namjoshi [7]. These results can be extended to allow the reduction of the parameterized synthesis problem to a distributed synthesis problem with a fixed number of components, which can in turn be solved by a modification of the bounded synthesis procedure of Finkbeiner and Schewe [9]. As experiments with the original, naïve implementation of parameterized synthesis revealed that only very small specifications could be handled, Khalimov et al. [14] introduced a number of optimizations that improved runtimes for synthesis of token-ring systems by several orders of magnitude. In this paper, we will show how the resulting synthesis method can serve as the basis for synthesizing an implementation for the parameterized AMBA AHB specification.

Contributions. We demonstrate how to synthesize a parameterized implementation of the AMBA AHB, with guaranteed correctness for any number of masters. To this end, we translate the LTL specification

${ }^{*}$ This work was supported by the Austrian Science Fund (FWF) under the RiSE National Research Network (S11406).

K. Chatterjee, R. Ehlers, and S. Jha (Eds.):

Third Workshop on Synthesis (SYNT 2014)

EPTCS 157, 2014, pp. $68-83$ doi 10.4204/EPTCS.157.9 (c) R. Bloem, S. Jacobs \& A. Khalimov

This work is licensed under the Creative Commons Attribution License. 
of the AMBA AHB (as found in [12]) into a version that is suitable for parameterized synthesis in token rings, and address several challenges with respect to theoretical applicability and practical feasibility:

1. We show how to localize global input and output signals, i.e., those that cannot be assigned to one particular master. This is necessary since our approach is based on the replication of components that act only on local information.

2. We introduce theoretical extensions of the cutoff results for token rings that support some of the features of the AMBA specification. This includes the handling of assumptions on local and global inputs, and of the fully asynchronous timing model (which includes the synchronous behavior intended in AMBA).

3. We show how to handle multiple process templates to support a special process with properties different from other processes.

4. We describe some further optimizations that make synthesis feasible, in particular based on the insight that the AMBA protocol features three different types of accesses, and the control structures for these accesses can be synthesized (to some degree) independently.

Finally, we report on our practical experience with parameterized synthesis of the AMBA protocol, pointing out weaknesses and open problems in current synthesis approaches.

\section{The AMBA Case Study}

ARMs Advanced Microcontroller Bus Architecture (AMBA) [2] is a communication bus for a number of masters and clients on a microchip. The most important part of AMBA is the Advanced Highperformance Bus (AHB), a system bus for the efficient connection of processors, memory, and devices.

The bus arbiter is the critical part of the AHB, ensuring that only one master accesses the bus at any time. Masters send HBUSREQ to the arbiter if they want access, and receive HGRANT if they are allowed to access it. Masters can also ask for different kinds of locked transfers that cannot be interrupted.

The exact arbitration protocol for AMBA is not specified. Our goal is to synthesize a protocol that guarantees safety and liveness properties. According to the specification, any device that is connected to the bus will react to an input $t$ with a delay of one time step. I.e., we are considering Moore machines. In the following, we introduce briefly which signals are used to realize the controller of this bus.

Requests and grants. The identifier of the master which is currently active is stored in the $n+1$-bit signal HMASTER[ $n: 0]$, with $n$ chosen such that the number of masters fits into $n+1$ bits. To request the bus, master i raises signal HBUSREQ[i]. The arbiter decides who will be granted the bus next by raising signal HGRANT[i]. When the client raises HREADY, the bus access starts at the next tick, and there is an update HMASTER $[n: 0]:=\mathrm{i}$, where HGRANT[i] is currently active.

Locks and bursts. A master can request a locked access by raising both HBUSREQ[i] and HLOCK[i]. In this case, the master additionally sets HBURST[1:0] to either SINGLE (single cycle access), BURST4 (four cycle burst) or INCR (unspecified length burst). For a BURST4 access, the bus is locked until the client has accepted 4 inputs from the master (each signaled by raising HREADY). In case of a INCR access, the bus is locked until HBUSREQ[i] is lowered. The arbiter raises HMASTLOCK if the bus is currently locked.

LTL specification. The original natural-language specification [2] has been translated into a formal specification in the GR(1) fragment of LTL before [12, 6, 10]. Figure 1 shows the environment assumptions and system guarantees from [12] that will be the basis for our parameterized specification. The full specification is $(A 1 \wedge \ldots \wedge A 4) \rightarrow(G 1 \wedge \ldots \wedge G 11)$. 


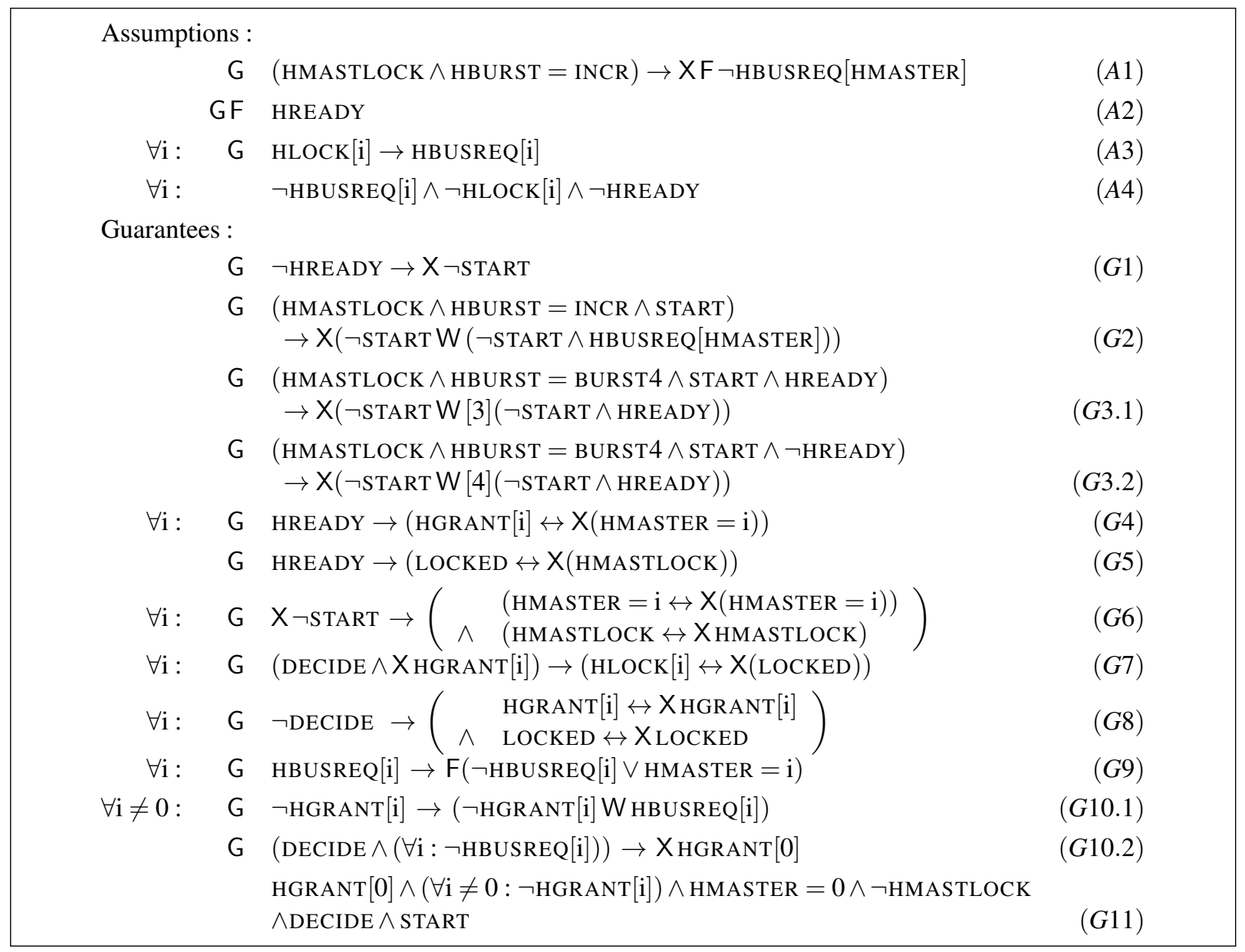

Figure 1: Formal specification of the AMBA AHB [12], in GR(1) fragment of LTL.

\section{Definitions}

A labeled transition system (LTS) over sets $O$ of output variables and I of input variables is a tuple $\left(Q, Q_{0}, \Sigma, \delta, \lambda\right)$ where $Q$ is the set of states, $Q_{0} \subseteq Q$ is the set of initial states, $\Sigma=2^{I}$ is the set of inputs (also called transition labels), $\delta \subseteq Q \times \Sigma \times Q$ is the transition relation, and $\lambda: Q \rightarrow 2^{O}$ is the output function (also called state-labeling function). Variables from $O \cup I$ will be used as atomic propositions in our specifications.

\subsection{System Model}

In this section we define the token ring system - the LTS that consists of replicated copies of a process connected in a uni-directional ring. Transitions in a token ring system are either internal or synchronized (in which one process sends the token to the next process along the ring). The token starts in a nondeterministically chosen process.

Fix a set $\mathrm{O}_{\mathrm{pr}}$ of (local) output variables that contain a distinguished output variable snd, and a set $\mathrm{I}_{\mathrm{pr}}$ of (local) input variables that contain a distinguished input variable rcv.

Process Template $P$. Let $\Sigma_{\mathrm{pr}}=2^{\mathrm{I}_{\mathrm{pr}}}$. A process template $P$ is a LTS $\left(Q, Q_{0}, \Sigma_{\mathrm{pr}}, \boldsymbol{\delta}, \boldsymbol{\lambda}\right)$ over $\mathrm{O}_{\mathrm{pr}}$ and: 
i) The state set $Q$ is finite and can be partitioned into two non-empty disjoint sets: $Q=T \cup N T$. States in $T$ are said to have the token.

ii) The initial state set is $Q_{0}=\left\{\boldsymbol{l}_{t}, \boldsymbol{l}_{n}\right\}$ for some $\boldsymbol{l}_{t} \in T, \boldsymbol{l}_{n} \in N T$.

iii) The output function $\lambda: Q \rightarrow 2^{\mathrm{O}_{\mathrm{pr}}}$ satisfies that for every $q \in N T$, snd $\notin \lambda(q)$.

iv) Every transition $q \stackrel{\text { in }}{\rightarrow} q^{\prime}$ with snd $\in \lambda(q)$ satisfies that $q$ has the token and $q^{\prime}$ does not.

v) Every transition $q \stackrel{\text { in }}{\rightarrow} q^{\prime}$ with $\operatorname{rcv} \in$ in satisfies that $q$ does not have the token and $q^{\prime}$ does.

vi) Every transition $q \stackrel{\text { in }}{\rightarrow} q^{\prime}$ with snd $\notin \lambda(q)$ and $\operatorname{rcv} \notin$ in satisfies that $q$ has the token if and only if $q^{\prime}$ has the token.

vii) The process template is non-terminating: for every $q \in N T$ and every in $\in \Sigma_{\mathrm{pr}}$ there exists $q \stackrel{\text { in }}{\rightarrow} q^{\prime}$; and for every $q \in T$ and every in $\in \Sigma_{\text {pr }}$ with $\operatorname{rcv} \notin$ in there exists $q \stackrel{\text { in }}{\rightarrow} q^{\prime}$.

$\dagger$ ) Consider a fairness condition $A_{l o c}$ over $\mathrm{I}_{\mathrm{pr}} \cup \mathrm{O}_{\mathrm{pr}}{ }_{1}^{1}$ From any state $q$ with the token, under any input sequence satisfying $A_{l o c}$, the process will reach a state $q^{\prime}$ where it sends the token. We call this requirement $\dagger$.

Ring Topology $R$. A ring is a directed graph $R=(V, E)$, where the set of vertices is $V=\{1, \ldots, k\}$ for some $k \in \mathbb{N}$, and the set of edges is $E=\{(i, i \oplus 1) \mid i \in V\}$. Vertices are called process indices.

Token-Ring System $P^{R}$. Fix a ring topology $R=(V, E)$. Let $\mathrm{I}_{\text {sys }}:=\left(\mathrm{I}_{l o c} \times V\right) \cup \mathrm{I}_{\text {glob }}$ be the system input variables, where local inputs $\mathrm{I}_{l o c}$ and global inputs $\mathrm{I}_{g l o b}$ are such that $\mathrm{I}_{\mathrm{pr}}=\mathrm{I}_{l o c} \cup \mathrm{I}_{g l o b}$. Define $\Sigma_{\text {sys }}=2^{\mathrm{I}_{\mathrm{sys}}}$. For system input in $\in \Sigma_{\text {sys }}$, let in $(v) \subseteq$ in denote the input to process $v$ (including global inputs). Let $\mathrm{O}_{\text {sys }}:=\mathrm{O}_{\mathrm{pr}} \times V$ be the system output variables. For $(p, i)$ in $\mathrm{O}_{\text {sys }}$ or in $\mathrm{I}_{\text {sys }} \backslash \mathrm{I}_{\text {glob }}$ we write $p_{i}$.

Given a process template $P=\left(Q, Q_{0}, \Sigma_{\mathrm{pr}}, \delta, \lambda\right)$ over $\mathrm{O}_{\mathrm{pr}}$ and $\mathrm{I}_{\mathrm{pr}}$ and a token ring topology $R=(V, E)$, define the token-ring system $P^{R}$ as the finite LTS $\left(S, S_{0}, \Sigma_{\text {sys }}, \Delta, \Lambda\right)$ over $\mathrm{O}_{\text {sys }}$ and $\mathrm{I}_{\text {sys }}$, where:

- The set $S$ of global states is $Q^{V}$, i.e., all functions from $V$ to $Q$. If $s \in Q^{V}$ is a global state then $s(i)$ denotes the local state of the process with index $i$.

- The set of global initial states $S_{0}$ contains all $s_{0} \in Q_{0}^{V}$ in which exactly one of the processes has the token.

- The labeling $\Lambda(s) \subseteq \mathrm{O}_{\text {sys }}$ for $s \in S$ is defined as follows: $p_{i} \in \Lambda(s)$ if and only if $p \in \lambda(s(i))$, for $p \in \mathrm{O}_{\mathrm{pr}}$ and $i \in V$.

Finally, define the global transition relation $\Delta$. In a fully asynchronous token ring, a subset of all processes can make a transition in each step of the system, i.e., $\Delta$ consists of the following set of transitions:

- An internal transition is an element $\left(s\right.$, in, $\left.s^{\prime}\right)$ of $S \times \Sigma_{\text {sys }} \times S$ for which there are process indices $M \subseteq V$ such that

i) for all $v \in M$ : snd $\notin \lambda(s(v))$ and $\operatorname{rcv} \notin \operatorname{in}(v)$,

ii) for all $v \in M: s(v) \stackrel{\operatorname{in}(\mathrm{v})}{\rightarrow} s^{\prime}(v)$ is a transition of $P$,

iii) for all $u \in V \backslash M: s(u)=s^{\prime}(u)$.

- A token-passing transition is an element $\left(s\right.$, in, $\left.s^{\prime}\right)$ of $S \times \Sigma_{\text {sys }} \times S$ for which there are process indices $M \subseteq V$ and two indices $v, w \in M$ such that $(v, w) \in E$ and

\footnotetext{
${ }^{1}$ Discussion of fairness conditions is deferred until Sect. 3.2
} 
i) snd $\in \lambda(s(v))$, and $\forall u \in M \backslash\{v\}$ : snd $\notin \lambda(s(u))$ - i.e., only process $v$ sends the token,

ii) $\operatorname{rcv} \in \operatorname{in}(w)$ and for all $u \in M \backslash\{w\}$ : $\operatorname{rcv} \notin \operatorname{in}(u)$ - i.e., only process $w$ receives the token,

iii) for every $u \in M: s(u) \stackrel{\operatorname{in}(\mathrm{u})}{\rightarrow} s^{\prime}(u)$ is a transition of $P$,

iv) for every $u \in V \backslash M: s^{\prime}(u)=s(u)$.

Special cases of the fully asynchronous token ring are the synchronous token ring and the interleaving token ring. In a synchronous token ring, $M=V$ for internal and token-passing transitions. I.e., always all processes make a transition simultaneously. In an interleaving token ring, $M=\{v\}$ for some $v \in V$ for internal transitions, and $M=\{v, w\}$ for $(v, w) \in E$ for token-passing transitions. I.e., at each moment either exactly one process makes an internal transition, or one process sends a token to the next process.

System Run. Fix a ring topology $R=(V, E)$. A run of a token ring system $P^{R}=\left(S, S_{0}, \Sigma_{\mathrm{sys}}, \Delta, \Lambda\right)$ over $\mathrm{O}_{\text {sys }}$ and $\mathrm{I}_{\text {sys }}$ is a finite or infinite sequence $x=\left(s_{1}, \mathrm{in}_{1}, M_{1}\right)\left(s_{2}, \mathrm{in}_{2}, M_{2}\right) \ldots$, where:

- $s_{1} \in S_{0}, s_{k} \in S$ and $\operatorname{in}_{k} \in \Sigma_{\text {sys }}$ for any $k \leq|x|$,

- for all $k<|x|:\left(s_{k}, \operatorname{in}_{k}, s_{k+1}\right) \in \Delta$,

- for all $k<|x|: M_{k}$ is the set of processes making a transition (see $M$ in the definition of $\Delta$ ).

\subsection{Parameterized Systems and Specifications}

The parameterized ring is the function $\mathscr{R}: n \mapsto \mathscr{R}(n)$, where $n \in \mathbb{N}$ and $\mathscr{R}(n)$ is the ring with $n$ vertices. A parameterized token ring system is a function $P^{\mathscr{R}}: n \mapsto P^{\mathscr{R}(n)}$, where $n \in \mathbb{N}$ and $P$ is a given process template. When necessary to disambiguate we explicitly write 'parameterized fully asynchronous token ring systems' or 'parameterized interleaving token ring systems'.

A parameterized specification is a sentence in indexed temporal logic, that is, a temporal logic formula with indexed variables and quantification over indices. Variables are from the set of output and input variables $\mathrm{O}_{\mathrm{pr}} \cup \mathrm{I}_{\mathrm{pr}}$, and indices refer to different copies of process templates. A parameterized token ring system $P^{\mathscr{R}}$ satisfies a parameterized specification $\phi$, written $P^{\mathscr{R}} \models \phi$, iff $\forall n$ : $P^{\mathscr{R}(n)} \models \phi$. This definition assumes a fixed semantics for temporal logic formulas in labeled transition systems. Below, we introduce a slightly non-standard semantics as a modification of the (action-based) semantics in Emerson and Namjoshi [7] to the case of open systems.

Semantics for Open Systems. Emerson and Namjoshi [7] consider closed systems (i.e., without inputs), but with transitions labeled by actions that can also be used in specifications. In the semantics defined below, we simulate an action a by an input corresponding to a. Furthermore, for defining when a given process satisfies a formula, we consider the projection of the run onto those points in time where the process actually makes a transition, just like actions are only considered when a transition fires.

In addition, we extend our semantics to the fully asynchronous timing model, which in particular includes the synchronous timing model that is needed for reasoning about the AMBA case study. 2 This leads to additional problems: the natural ways to extend the semantics to properties of more than one process is to consider either the projection to those points in time where at least one of the processes makes a step, or to those where all processes make a step. Both cases are undesirable: in the first case, we also consider inputs that the processes cannot read, and in the second case the property will

\footnotetext{
${ }^{2}$ Note that in the synchronous timing model, our semantics is the same as the standard semantics (every process always makes a transition, so all inputs are considered), but we need the fully asynchronous case for the cutoff result in Thm. 3 Intuitively, in synchronous systems an implementation can count the number of global steps until it receives the token again, and therefore correctness of such implementations may depend on the size of the ring, making cutoff results impossible. Thus, systems that are correct in the synchronous but not in the fully asynchronous case are of limited interest to us.
} 
only be guaranteed at those points in time where all processes make a step together - which is clearly undesirable, e.g., for a mutual exclusion property. Therefore, in properties that talk about more than one process, we do not allow input signals at all.

Fix a token ring system $P^{R}=\left(S, S_{0}, \Sigma_{\text {sys }}, \Delta, \Lambda\right)$ over $\mathrm{O}_{\text {sys }}$ and $\mathrm{I}_{\text {sys. }}$. We describe the semantics of parameterized properties 1-indexed properties over $\mathrm{O}_{\text {sys }}$ and $\mathrm{I}_{\text {sys }}$, and 2-indexed properties over $\mathrm{O}_{\text {sys }}$.

Semantics of 1-indexed properties. 1 -indexed properties are of the form $\forall i . \varphi(i)$, where $\varphi(i)$ is an LTL formula over system variables that are indexed with $i$.

Fix a process index $j$. Given a run $\left(s_{1}, \mathrm{in}_{1}, M_{1}\right)\left(s_{2}, \mathrm{in}_{2}, M_{2}\right) \ldots$, consider the sub-sequence that contains exactly those $\left(s_{k}, \operatorname{in}_{k}, M_{k}\right)$ with $j \in M_{k}$. The local run of process $j$ is obtained by mapping each $\left(s_{k}, \operatorname{in}_{k}, M_{k}\right)$ in the sub-sequence to $\left(s_{k}(j), \operatorname{in}_{k}(j)\right)$, where $s_{k}(j)$ is the local state of $j$ and $\operatorname{in}_{k}(j)$ are inputs to $j$. Then, a system run satisfies $\varphi(j)$ iff the local run of process $j$ satisfies $\varphi(j)$. The latter satisfaction is defined in the usual way. Note that since we consider only elements of the system run where process $j$ makes a step, we can use the next-time operator X (interpreted locally, cp. [14, Sect. 5.2][7, Sect. 2.5]).

Example. Consider a typical 1-indexed property of an arbiter $\forall i . \mathrm{G}\left(r_{i} \rightarrow \mathrm{F} g_{i}\right)$ ('for every process, every request should be finally granted'). In the semantics of 1 -indexed properties described above, this property should be read as: 'for every process, every request that has been seen by the process should be finally granted'. Another example: in the new semantics the property $\forall i . \mathrm{G}\left(r_{i} \rightarrow \mathrm{X} g_{i}\right)$ should be read as 'for every process, every request that has been seen by the process should be granted the next step'. Notice that the environment cannot falsify the property by not scheduling the process.

Semantics of 2-indexed properties. 2-indexed properties are of the form $\forall i, j . \varphi(i, j)$, where $\varphi(i, j)$ is an $\mathrm{LTL} \backslash \mathrm{X}$ formula over output variables (and no input variables) that are indexed with $i$ or $j$. Satisfaction for fixed process indices $i, j$ is defined in the standard way: A system run $\left(s_{1}, \mathrm{in}_{1}, M_{1}\right)\left(s_{2}, \mathrm{in}_{2}, M_{2}\right) \ldots$ satisfies $\varphi(i, j)$ iff the sequence $\Lambda\left(s_{1}\right) \Lambda\left(s_{2}\right) \ldots$ satisfies $\varphi(i, j)$. I.e., we consider all elements of the system run, no matter if processes $i$ and $j$ make the transition or not.

Example. Consider a typical 2-indexed property of the mutual exclusion $\forall i \neq j . G \neg\left(g_{i} \wedge g_{j}\right)$. In the semantics of 2-indexed properties described above, the property should be read in a usual way: 'it is never the case that two processes grant at the same time.

Semantics of $A_{\forall i . a s s(i)} \varphi$. Let $\varphi$ be a 1- or 2-indexed property as introduced above. A system satisfies $\mathrm{A}_{\forall i \text {.ass }(i)} \varphi$ iff any system run that satisfies $\forall i$.ass $(i)$ also satisfies $\varphi$, where satisfaction of 1-indexed $\forall i$.ass $(i)$ and of $\varphi$ as defined above.

Note on the Semantics of GR(1). The AMBA specification [12] is defined in the GR(1) fragment of LTL, where the implication between assumptions and guarantees is usually interpreted with a special semantics [15]. In this paper, we instead use the standard semantics for this implication.

\subsection{Parameterized Synthesis Problem}

The parameterized synthesis problem in token rings is: given a parameterized specification $\varphi$, find an implementation $P$ such that $P^{\mathscr{R}} \models \varphi$. The problem is in general undecidable:

Theorem 1 ([11], Theorem 3.5). The parameterized synthesis problem of interleaving token rings with no global inputs is undecidable for specifications $\forall i, j . \mathrm{A} \varphi(i, j)$, where $\mathrm{A} \varphi(i, j)$ is an $L T L \backslash X$ formula over processes $i, j$.

The proof reduces the undecidable problem of distributed synchronous synthesis [17] to distributed synthesis of an interleaving token ring of size 2, which implies undecidability of parameterized synthesis.

Note that Theorem 1 does not apply to specifications of the form $A_{\forall i . a s s(i)} \forall j . \varphi(j)$. In fact, we can use the hub-abstraction technique (see [14, Section 6]) to prove the following: 
Observation 1. The parameterized synthesis problem of token rings without global inputs is decidable for specifications $\forall j . \mathrm{A}_{\forall i \text { ass }(i)} \varphi(j)$ where ass $(i)$ and $\varphi(i)$ are $L T L$ formulas over process $i$.

\section{The Existing Parameterized Synthesis Approach}

The parameterized synthesis problem in token rings is in general undecidable, but Jacobs and Bloem [11] have introduced a semi-decision procedure for the problem. It is based on i) the cutoff results of [7], which state that model checking parameterized token rings is equivalent to model checking token rings of a cutoff size, and ii) the bounded synthesis method [9] that turns an undecidable synthesis problem (of synthesizing a token ring of fixed size) into a possibly infinite sequence of decidable synthesis problems (of synthesizing a token ring of fixed size in which a process implementation is not larger than the bound) by iterative bounding the size of process implementations.

\subsection{Cutoff Results in Token Rings}

A cutoff for a parameterized specification $\varphi$ is a number $c \in \mathbb{N}$ s.t. $P^{\mathscr{R}(c)} \models \varphi \Longleftrightarrow \forall n \geq c: P^{\mathscr{R}(n)} \models \varphi$.

Theorem 2 ([7], Theorem 3). For interleaving parameterized token ring systems with no global inputs and parameterized specifications $\forall i . \mathrm{A}_{\forall i \text {.ass }(i)} \varphi(i)$, where $\varphi(i)$ and ass $(i)$ are $L T L$ formulas over process $i$, the cutoff is 2 .

Corollary 1 ([11]). The parameterized synthesis problem of interleaving token rings with no global inputs for parameterized specifications $\forall i . A_{\forall i . a s s(i)} \varphi(i)$, where $\varphi(i)$ and ass $(i)$ are $L T L$ formulas over process $i$, can be reduced to the synthesis problem of the token ring of size 2 .

\subsection{Bounded Synthesis Method}

By bounding the desired size of implementations, bounded synthesis [9] reduces the synthesis problem to a sequence of SMT problems. Uninterpreted functions are used to describe the transition relation, output functions, and auxiliary 'ranking' functions, and the SMT solver tries to find valuations of these functions such that the specification is satisfied. The flow is the following:

1. Automata translation: The negation of a given specification $\varphi$ is translated into a non-deterministic Büchi automaton $A_{\neg \varphi}$.

2. SMT encoding: We encode a ranking function $\rho$ on states of the product of the specification automaton $A_{\neg \varphi}$ and the uninterpreted system. Consider a transition from composed state $(q, s)$ to $\left(q^{\prime}, s^{\prime}\right)$, where $q, q^{\prime}$ are states of $A_{\neg \varphi}$ and $s, s^{\prime}$ are states of the system. Then we require $\rho\left(q^{\prime}, s^{\prime}\right)>$ $\rho(q, s)$ if $q^{\prime}$ is an accepting state of $A_{\neg \varphi}$, and otherwise $\rho\left(q^{\prime}, s^{\prime}\right) \geq \rho(q, s)$. The rule ensures that the SMT constraints are satisfiable if and only if the product does not have loops with an accepting state of the automaton, and a solution represents a correct implementation of the system.

3. SMT solving, iteration for increasing bounds: If the SMT constraints in step 2 are satisfiable, then return the implementation. Otherwise, there exists no implementation of the given size bound; we increase the bound and repeat step 2.

For the details of the SMT encoding that we use for synthesis of token rings see Khalimov et al. [14]. 


\section{Challenges for the Existing Approach}

Based on the existing approach for parameterized synthesis, we want to synthesize a system that satisfies

$$
\mathrm{A}((A 1 \wedge \ldots \wedge A 4 \wedge \text { FairSched }) \rightarrow(G 1 \wedge \ldots \wedge G 11 \wedge T R))
$$

where

- FairSched is the form $\forall i$. GF $s c h_{i}$, specifying that every process is scheduled infinitely often.

- $T R$ are guarantees ensuring that the process template satisfies the requirements of the token ring process template defined in Sect. 3.1.

$$
\begin{array}{ll}
\forall i . & \mathrm{G}(\operatorname{SEND}[\mathrm{i}] \rightarrow \operatorname{TOK}[\mathrm{i}]) \\
\forall i . & \mathrm{G}(\mathrm{TOK}[\mathrm{i}] \wedge \neg \operatorname{SEND}[\mathrm{i}] \rightarrow \mathrm{XTOK}[\mathrm{i}]) \\
\forall i . & \mathrm{G}(\neg \mathrm{TOK}[\mathrm{i}] \wedge \neg \operatorname{SEND}[i-1] \rightarrow \mathrm{X} \neg \mathrm{TOK}[\mathrm{i}]) \\
\forall i . & \mathrm{G}(\operatorname{TOK}[\mathrm{i}] \rightarrow \operatorname{FSEND}[\mathrm{i}])
\end{array}
$$

As the existing cutoff results of Emerson and Namjoshi [7] (or their extensions by Aminof et al. [1]) do not support all features of the AMBA specification, we need to address the following challenges:

1. Synchronous AMBA and global inputs: The AMBA protocol uses synchronous timing and has several global inputs (that are shared between all processes), while the cutoff results in [7, 1] are for interleaving systems with local action labels instead of inputs. We have discussed in Sect. 3.2 how actions simulate local inputs, but global inputs are not supported in the existing cutoff theorems.

In Sect. 6.1 we extend the cutoff results to fully asynchronous token rings with global inputs. For our synthesis approach, we will use the fact that correctness of an implementation in the fully asynchronous case implies correctness in the synchronous case.

2. Global outputs: The AMBA specification assumes that there are global outputs, i.e., those that depend on the global state of the system, such as HMASTLOCK. This is not handled by [7, 1], and in Sect. 6.2 we address this by synthesizing local outputs that can be manually converted to suitable global outputs with simple logical operations.

3. Special 0-process, immediate reaction, global information: The AMBA specification distinguishes between master number 0 and all other masters. We support this by synthesizing two different process implementations, one that serves master 0 , and one for all other processes. Furthermore, process 0 is supposed to immediately grant master 0 when no process receives a HBUSREQ[i] signal - this is a problem since only processes that have the token should give a grant, and information about requests of other processes is not available to process 0 . We show how to handle this by weakening the specification and introducing an auxiliary global input in Sect. 6.3 .

\section{Obtaining and Handling a Parameterized AMBA Specification}

In the following, we will show how we obtained a parameterized AMBA specification suitable to our parameterized synthesis approach, and how we extended the approach to handle this specification.

\subsection{Addressing Challenges 'Synchronous AMBA' and 'Global Inputs'}

We will first extend the cutoff results for token rings to fully asynchronous systems with global inputs, for restricted classes of process templates and assumptions. Since these classes are not sufficient to model AMBA, we will afterwards introduce a method to localize assumptions in a sound but incomplete way. 


\subsubsection{Complete Approach: New Cutoff Results}

We consider systems and specifications that satisfy the following assumptions:

a) $P=\left(Q, Q_{0}, \Sigma_{\mathrm{pr}}, \delta, \lambda\right)$ is such that: $\forall q \in Q$ with snd $\in \lambda(q)$ there exists unique $q^{\prime} \in Q$ such that $q \stackrel{\text { in }}{\rightarrow} q^{\prime}$ for any input in $\in \Sigma_{\text {pr }}$. I.e., in all sending states the process ignores inputs.

b) The assumptions $\forall i . a s s(i)$ are of the form $\forall i . G \alpha(i)$ or of the form $\forall i . \alpha(i)$, where $\alpha(i)$ is a Boolean formula over inputs (including global inputs) of process $i$.

Then, we can prove the following theorem:

Theorem 3. Assume conditions (a) and (b). Then, for parameterized fully asynchronous token ring systems and parameterized specifications as stated below, the cutoffs are:

- for $\forall i . \mathrm{A}_{\forall i \mathrm{G} \alpha(i)} \varphi(i)$ the cutoff is 2 ,

- for $\forall i, j . \mathrm{A}_{\forall i \mathrm{G} \alpha(i)} \psi(i, j)$ the cutoff is 4 ,

where $\alpha(i)$ is a Boolean formula over inputs of process $i, \varphi(i)$ is an $L T L$ formula over inputs and outputs of process $i$, and $\psi(i, j)$ is an $L T L \backslash X$ formula over outputs of processes $i, j$.

Note that the problem becomes undecidable if we do not restrict fair path properties, i.e., if we remove (b) but still assume (a):

Observation 2. The parameterized model checking problem for fully asynchronous token rings with global inputs and properties of the form $\forall i$. $_{\forall i . a s s(i)} \varphi(i)$ is undecidable, where ass $(i)$ and $\varphi(i)$ are $L T L$ formulas over inputs and outputs of process $i$ (including global inputs).

Proofs for Theorem 3 and Observation 2 can be found in the full version of the paper [5]. Note that Theorem 3 does not support all assumptions in the AMBA specification (Fig. 1): A3 and A4 are supported by the theorem, but A1 and A2 are not.

\subsubsection{Incomplete Approach: Localization of Assumptions}

Since Theorem 3 does not support assumptions A1 and A2, we introduce an approach that localizes the assumptions, essentially rewriting the specification $\forall j . \mathrm{A}_{\forall i \text {. ass }(i)} \varphi(j)$ into a form $\left.\forall j . \mathrm{A}(\operatorname{ass}(j) \rightarrow \varphi(j))\right\}^{3}$

However, this naive form of localization strengthens the AMBA specification too much, making it unrealizable. Instead, we use a specialized way for localizing assumptions in token rings.

Localization of assumptions in token rings. As suggested in [14, Sect.6],

$$
\mathrm{A}_{\forall i . \operatorname{ass}(i)} \forall j .(\operatorname{gua}(j) \wedge T R(j))
$$

is localized into

$$
\forall i . \mathrm{A}(\operatorname{ass}(i) \rightarrow T R(i)) \wedge(\operatorname{ass}(i) \wedge \mathrm{GF} \mathrm{TOK}[\mathrm{i}] \rightarrow \operatorname{gua}(i)),
$$

where $\operatorname{ass}(i)$ includes FairSched, and $T R$ are the token ring properties as defined in Sect. 5 .

This restores realizability in our case. Intuitively, this specification guarantees that $T R$ will be satisfied under the given local assumptions, and for the rest of the guarantees we can then assume that all other processes will eventually send the token, thus satisfying the additional assumption GF TOK $[\mathrm{i}]$.

Linking token possession to mutual exclusion. In addition to global assumptions, the original specification contains an implicit mutual exclusion property: G4 defines how HMASTER is updated by the

\footnotetext{
${ }^{3}$ Note that in some cases, the localized version is equivalent to the original one, e.g. for A2, since HREADY is a global input.
} 
HGRANT[i] signals. Note that G4 can only be satisfied if the HGRANT[i] are mutually exclusive. Since we know that the token can (and must) be used to ensure mutual exclusion, we explicitly specify this by adding G12: $\forall i$. HGRANT[i] $\rightarrow$ TOK[i]. Together with localization of assumptions, this ensures that the parameterized specification will be 1-indexed.

Resulting specification. The resulting specification is of the form

$$
\forall i . \mathrm{A}((\operatorname{ass}(i) \rightarrow T R(i)) \wedge((\operatorname{ass}(i) \wedge \mathrm{GF} \mathrm{TOK}[\mathrm{i}]) \rightarrow \operatorname{gua}(i) \wedge G 12)),
$$

i.e., a 1-indexed LTL property in prenex-indexed form.

While Theorem 3 supports some formulas of the type $A_{\forall i . a s s(i)} \forall j . g u a(j)$, solving the synthesis problem for formulas with assumptions in this form is costly. In particular, every liveness assumption introduces a loop (with length equal to the size of the ring under consideration) for every liveness guarantee in the specification. This severely blows up the size of the specification automaton. Thus, even for the liveness assumptions A3 and A4 that are supported by the theorem, we use the localization approach.

\subsection{Addressing Challenge 'Global Outputs'}

To address this challenge we define what is a localizable global output, introduce a special version of localizable global outputs we use for AMBA, and modify the specification to handle these global outputs.

Linking global to local outputs. For a given parameterized system, a localizable global output is a global output that can be expressed as a propositional formula over terms of the form $\forall i . \alpha(i)$ and $\exists i . \alpha(i)$, where $\alpha(i)$ is a propositional formula over outputs of process $i$.

Fixed solution for AMBA. The AMBA specification in Fig. 1 has global outputs HMASTLOCK, START, DECIDE, and HMASTER. We restrict synthesis to search for a solution with a fixed localizable implementation of global outputs, namely: For each global output signal $g$ we introduce a local output signal $g_{i}$, and define

- HMASTER $:=i$ whenever HMASTER $[\mathrm{i}]$ is high, and

- $g:=\exists i$. TOK $[\mathrm{i}] \wedge g_{i}$ for all other global outputs $g$.

Modification of the parameterized specification. According to the two previous steps, we should replace all global outputs in the specification with their specialized localizable definitions in terms of the new local outputs. For example, START should be replaced by $\exists i$.TOK[i] $\operatorname{START}[\mathrm{i}]$. However, in token ring systems the only communication between processes is token passing, and hence the value of $\exists i$.TOK $[\mathrm{i}] \wedge \mathrm{START}[\mathrm{i}]$ is not known to a process, except when it has the token (and thus defines that value). Thus, we replace each global output with its local version, e.g., START is replaced by START[i].

Note that the limited communication interface (via token passing) does not make AMBA unrealizable, even though processes cannot access the value of global outputs when they do not possess. Intuitively, this is because the token is the shared resource that guarantees mutual exclusion of grants, and therefore the values of these global signals should always be controlled by the process that has the token. In particular, outputs DECIDE and START are signals that are used to decide when to raise a grant and when to start and end a bus access ${ }^{4}$, which should only be done when the token is present. Similarly, signals HMASTLOCK and HMASTER should be controlled by the process that currently controls the bus (and hence has the token). By using only the local version of these signals in the specification, we force the implementation to never raise them unless the process has the token.

\footnotetext{
${ }^{4}$ The original AMBA specification [2] does not have these signals - they were introduced to simplify the formalization of the specification [12].
} 


\subsection{Addressing Challenges 'Special 0-process' and 'Global Information'}

The AMBA specification is of the form $A_{\forall i . a s s(i)}(\forall i \neq 0 . \varphi(i) \wedge \psi(0))$, i.e., it distinguishes the behavior of process 0. Recall the AMBA guarantees G10 from Fig. 1 (after localization steps of the previous sections):

$$
\begin{array}{rll}
\forall \mathrm{i} \neq 0: & \mathrm{G} & (\neg \text { HGRAnt }[\mathrm{i}] \rightarrow(\neg \text { HGRAnt }[\mathrm{i}] \text { W hbUSREQ }[\mathrm{i}]) \\
& \mathrm{G} \quad(\operatorname{DECIDE}[0] \wedge(\forall \mathrm{i}: \neg \text { HBUSREQ }[\mathrm{i}])) \rightarrow \text { X HGRAnt }[0]
\end{array}
$$

The distinction between 0 - and non-0-processes, as well as the required properties, present several additional challenges to the parameterized synthesis approach.

Distinguished 0-process. The process templates for 0- and non-0-processes for specifications of the form $\mathrm{A}_{\forall i . a s s(i)}(\forall i \neq 0 . \varphi(i) \wedge \psi(0))$ can be synthesized separately, i.e., first, synthesize a process template $P_{\varphi}$ for $\mathrm{A}_{\forall i . a s s(i)} \forall i . \varphi(i)$ and a process template $P_{\psi}$ for $\mathrm{A}_{\forall i . a s s(i)} \forall i . \psi(i)$. Then a combined token ring consisting of any number of copies of $P_{\varphi}$ and of one copy of $P_{\psi}$ at 0 vertex will satisfy $A_{\forall i . a s s(i)}(\forall i \neq 0 . \varphi(i) \wedge \psi(0))$. Hence we introduce a separate specification for the 0-process and synthesize it separately.

To this end, we also separate G11 into two parts, G11.1: $\neg$ HGRANT[i] $\wedge \neg$ HMASTLOCK[i] (for non0-processes) and G11.2: TOK $[0] \rightarrow \operatorname{HGRANT}[0] \wedge \operatorname{HMASTER}[0] \wedge \neg \operatorname{HMASTLOCK}[0]$ (for 0-process).

Immediate reaction. Guarantee G10.2 requires an immediate reaction to a state where no process receives a bus request. This is unrealizable for AMBA in token rings because mutual exclusion of the grants requires possession of the token and implies G(HGRANT[i] $\rightarrow$ TOK $[\mathrm{i}])$. To allow the process to wait for the token and then immediately react, we modify G10.2 to G (DECIDE $[0] \wedge(\forall \mathrm{i}: \neg \operatorname{HBUSREQ}[\mathrm{i}]) \wedge$ $\left.\left.\mathrm{X}_{\mathrm{TOK}}[0]\right) \rightarrow \mathrm{X}_{\text {HGRANT}}[0]\right)$.

Global information. G10.2 contains an index quantifier $\forall i$ inside the temporal operator G, which is not supported by Thm. 3. Intuitively, G10.2 requires 0-process to have global information about inputs of all processes, as it needs to react to a situation where HBUSREQ[i] is low for all $\mathrm{i}$. This is not possible when only HBUSREQ[0] is available as an input, so we introduce an auxiliary (global) input NO_REQ, and add the assumption $\forall i$.G(HBUSREQ $[\mathrm{i}] \rightarrow \neg$ NO_REQ). Then G10.2 becomes: G (DECIDE $[0] \wedge$ NO_REQ $\wedge$ $\mathrm{X}$ TOK $\left.[0]) \rightarrow \mathrm{X}_{\text {HGRANT }}[0]\right)$. Such guarantees and assumptions are allowed by Thm. 3 .

\subsection{Resulting Parameterized AMBA Specification}

We obtained the new specification from the one in Fig. 1 by localization of global assumptions (Sect.6.1), localization of global output signals HMASTER, HMASTLOCK, DECIDE, and START (Sect. 6.2), and separation of specifications for 0 - and non-0-processes (Sect. 6.3). The resulting assumptions and guarantees for non-0-processes are given in Fig. 2, the modifications for the 0-process in Fig. 3. The specifications to be synthesized are

$$
\begin{aligned}
& \forall i . \mathrm{A}((A 1 \wedge \ldots \wedge A 4 \rightarrow T R(i)) \wedge(A 1 \wedge \ldots \wedge A 5 \rightarrow G 1 \wedge \ldots \wedge G 10.1 \wedge G 11.1 \wedge G 12)), \text { and } \\
& \forall i . \mathrm{A}((A 1 \wedge \ldots \wedge A 4 \wedge A 6 \rightarrow T R(i)) \wedge(A 1 \wedge \ldots \wedge A 6 \rightarrow G 1 \wedge \ldots \wedge G 10.2 \wedge G 11.2 \wedge G 12)) .
\end{aligned}
$$

\section{Optimizations and Experiments}

In this section, we describe optimizations that proved to be crucial for the synthesis of the parameterized AMBA AHB, and present the results of parameterized synthesis in form of runtimes and resulting component implementations. 
Local Assumptions :

G $\quad((\operatorname{HMASTLOCK}[\mathrm{i}] \wedge(\operatorname{HBURST}=\mathrm{INCR}) \wedge \operatorname{HMASTER}[\mathrm{i}]) \rightarrow \mathrm{XF} \neg$ HBUSREQ$[\mathrm{i}])$

G FHREADY

G HLOCK[i] $\rightarrow$ HBUSREQ $[\mathrm{i}]$

$\neg$ HBUSREQ $[\mathrm{i}] \wedge \neg \mathrm{HLOCK}[\mathrm{i}] \wedge \neg \mathrm{HREADY}$

G F TOK[i]

Local Guarantees :

$$
\begin{aligned}
& \mathrm{G} \quad \neg \text { HREADY } \rightarrow \mathrm{X} \neg \text { START }[\mathrm{i}] \\
& \text { G } \quad(\text { HMASTLOCK }[\mathrm{i}] \wedge \text { HBURST }=\mathrm{INCR} \wedge \operatorname{START}[\mathrm{i}]) \\
& \rightarrow \mathrm{X}(\neg \operatorname{START}[\mathrm{i}] \mathrm{W}(\neg \operatorname{START}[\mathrm{i}] \wedge \operatorname{HBUSREQ}[\mathrm{i}])) \\
& \text { G } \quad(\text { HMASTLOCK }[\mathrm{i}] \wedge \text { HBURST }=\text { BURST } 4 \wedge \text { START }[\mathrm{i}] \wedge \text { HREADY }) \\
& \rightarrow \mathrm{X}(\neg \operatorname{START}[\mathrm{i}] \mathrm{W}[3](\neg \operatorname{START}[\mathrm{i}] \wedge \text { HREADY })) \\
& \text { G } \quad(\text { HMASTLOCK }[\mathrm{i}] \wedge \text { HBURST }=\text { BURST } 4 \wedge \operatorname{START}[\mathrm{i}] \wedge \neg \text { HREADY }) \\
& \rightarrow \mathrm{X}(\neg \mathrm{START}[\mathrm{i}] \mathrm{W}[4](\neg \mathrm{START}[\mathrm{i}] \wedge \text { HREADY })) \\
& \text { G } \quad \text { HREADY } \rightarrow(\text { HGRANT }[\mathrm{i}] \leftrightarrow \text { X HMASTER }[\mathrm{i}]) \\
& \text { G HREAdY } \rightarrow(\text { LOCKED }[\mathrm{i}] \leftrightarrow \text { X hMastlock }[\mathrm{i}])
\end{aligned}
$$

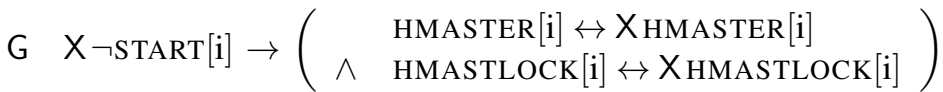

$$
\begin{aligned}
& \text { G } \quad(\text { DECIDE }[i] \wedge \text { X HGRANT }[\mathrm{i}]) \rightarrow\left(\text { HLOCK }[\mathrm{i}] \leftrightarrow \mathrm{X}_{\text {LOCKed }}[\mathrm{i}]\right) \\
& \mathrm{G} \quad \neg \text { DeCide }[\mathrm{i}] \rightarrow\left(\begin{array}{l}
\text { Hgrant }[\mathrm{i}] \leftrightarrow \mathrm{X}_{\text {Hgrant }}[\mathrm{i}] \\
\wedge \quad \text { LOCKed }[\mathrm{i}] \leftrightarrow \mathrm{X}_{\text {LOCKed }}[\mathrm{i}]
\end{array}\right) \\
& \text { G HBUSREQ }[\mathrm{i}] \rightarrow \mathrm{F}(\neg \operatorname{HBUSREQ}[\mathrm{i}] \vee \operatorname{HMASTER}[\mathrm{i}]) \\
& \text { G } \neg \text { HGRANT }[\mathrm{i}] \rightarrow(\neg \text { HGRANT }[\mathrm{i}] \text { W HBUSREQ }[\mathrm{i}]) \\
& \neg \text { HGRANT }[\mathrm{i}] \wedge \neg \text { HMASTLOCK }[\mathrm{i}] \\
& \text { G HGRANT[i] } \rightarrow \text { TOK }[\mathrm{i}]
\end{aligned}
$$

Figure 2: Parameterized AMBA specification for non-0-processes. G10.2 is only needed for 0-process.

Local Assumptions : as before: $\quad A 1, A 2, A 3, A 4, A 5$

new: $\quad$ G HBUSREQ $[\mathrm{i}] \rightarrow \neg$ NO_REQ

Local Guarantees : $\quad$ as before: $\quad G 1, G 2, G 3, G 4, G 5, G 6, G 7, G 8, G 9, G 12$

removed: $\quad G 10.1, G 11.1$

new: $\quad \mathrm{G}($ NO_REQ $\wedge \neg \mathrm{TOK}[0] \wedge \mathrm{X}$ TOK $[0]) \rightarrow$ X HGRANT $[0])$

modified: $\quad$ TOK $[0] \rightarrow$ HGRANT $[0] \wedge \operatorname{HMASTER}[0] \wedge \neg$ HMASTLOCK $[0]$

Figure 3: Parameterized AMBA specification for 0-process: modifications wrt. non-0-processes. 
Prototype. The basis of our experiments is PARTY, a tool for parameterized synthesis of token rings [13]. PARTY is written in Python, uses LTL3BA [3] for automata translation and Z3 [16] for SMT solving. All experiments were done on a x86_64 machine with 2.60GHz, 12GB RAM. Prototype implementation and specification files can be found at https://github.com/5nizza/Party/(branch 'amba-gr1').

Synchronous Hub Abstraction [14, Sect.6]. Synchronous hub abstraction can be applied to 1-indexed specifications. It lets the environment simulate all but one process, and always schedules this process. Thus, the synthesizer searches for a process template in synchronous setting with additional assumptions on the environment, namely: i) the environment sends the token to the process infinitely often, and ii) the environment never sends the token to the process if it already has it. Note that synchronous hub abstraction is sound and complete for the semantics of 1-indexed properties introduced in Sect. 3.2. Also note that after applying this optimization any monolithic synthesis method can be applied to the resulting specification in Sect. 6.4

Hardcoding States With and Without the Token [14, Sect.4]. The number of states with and without the token in a process template defines the degree of the parallelism in a token ring. Parallelism increases with the number of states that do not have the token. In the AMBA case study, any action with grant depends on having the token. Thus we divide states into one that does not have the token, and all others that have the token, by hardcoding the TOK $[\mathrm{i}]$ output function.

Decompositional Synthesis of Different Grant Schemes. The idea of the decompositional synthesis is: synthesize a subset of the properties, then synthesize a larger subset using the model from the previous step as basis. Consider an example of the synthesis of the non-0-process of AMBA. The flow is:

1. Assume that every request is locked with BURST4, i.e., add the assumption $\mathrm{G}(\mathrm{HLOCK}[\mathrm{i}] \wedge \mathrm{HBURST}=$ BURST4) to the specification. This implicitly removes guarantee G2 and assumption A1 from the specification. Synthesize the model. The resulting model has 10 states (states $t 0, . ., t 9$ and transitions between them in Fig. 47.

2. Use the model found in the previous step as a basis: assert the number of states, values of output functions in these states, transitions for inputs that satisfy the previous assumption. Transitions for inputs that violate the assumption from step 1 are not asserted, and thus left to be synthesized.

Now relax the assumptions: allow locked and non-locked BURST4 requests, i.e., replace the previous assumption with $\mathrm{G}($ HBURST $=$ BURST4). Again, this implicitly removes $\mathrm{G} 2$ and A1. In contrast to the last step, now guarantee G3 is not necessarily 'activated' if there is a request.

Synthesize the model. This may require increasing the number of states (and it does in the case of non-0 process ) - add new states and keep assertions on all the previous states.

3. Assert the transitions of the model found, like in the previous step.

Remove all added assumptions and consider the original specification. Synthesize the final model.

Although for AMBA this approach was successful, it is not clear how general it is. For example, it does not work if we start with locked BURST4 and HREADY always high, and then try to relax it. Also, the separation into sets of properties to be synthesized was done manually.

Optimization of SMT Encoding. Recall from Sect. 4 that SMT based bounded synthesis, given an automaton $A_{\neg \varphi}$ of the negation of specification $\varphi$ and an unknown process template $P=\left(Q_{P}, Q_{0}, \Sigma_{\mathrm{pr}}, \delta_{P}\right.$, out $)$ with a fixed number of states, encodes the product automaton $A_{\neg \varphi} \times P$ into SMT constraints such that $A_{\neg \varphi} \times P$ contains no reachable loops with an accepting state of the $A_{\neg \varphi}$ iff SMT constraints are satisfiable. Below is a general assertion from which the SMT query is composed:

$$
\bigwedge q \in Q_{P} \bigwedge a \stackrel{\mathrm{i}, \mathrm{o}}{\rightarrow} b \in \delta_{A_{\neg \varphi}}: \quad \rho(a, q) \geq 0 \wedge o=\operatorname{out}(q) \rightarrow \rho\left(b, \delta_{P}(q, i)\right) \triangleright \rho(a, q)
$$




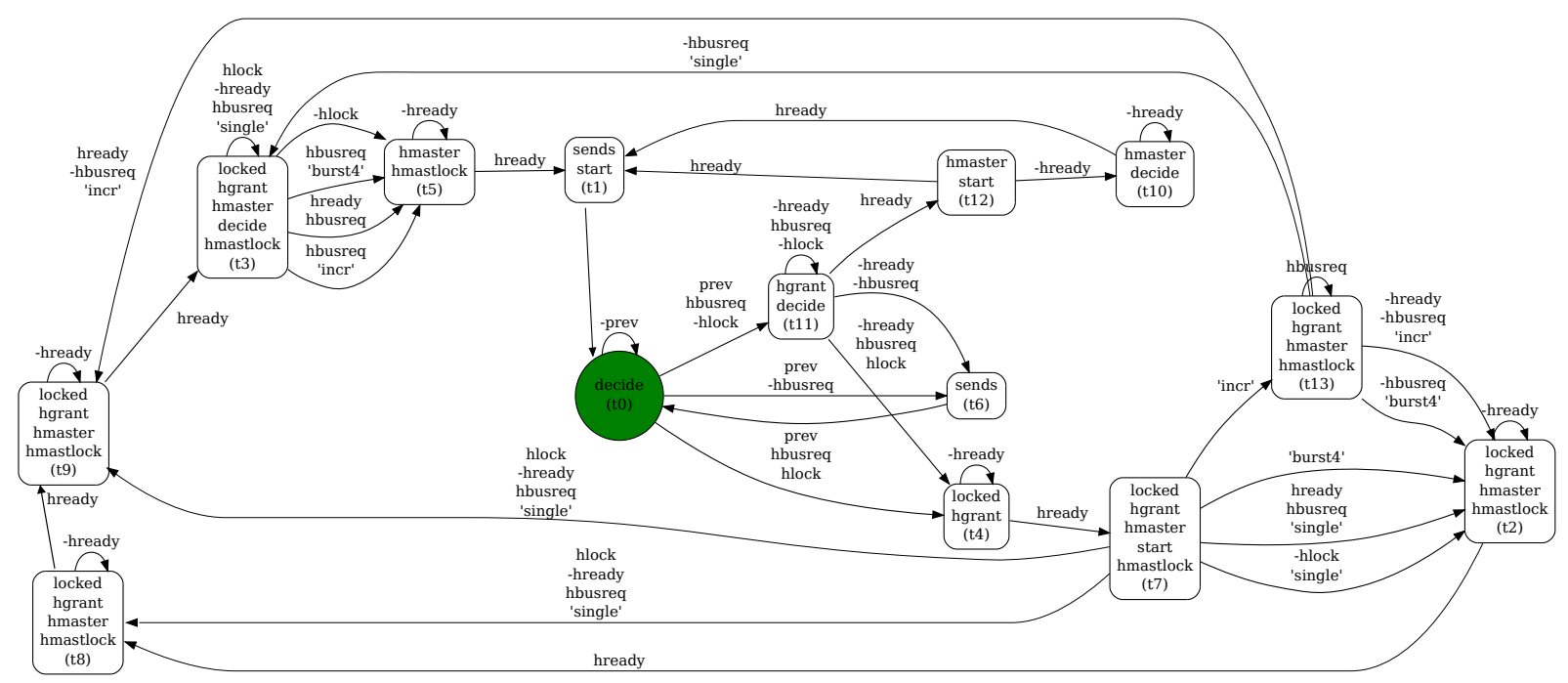

Figure 4: Synthesized model of non-0-processes. Circle green state $(t 0)$ is without the token, other states are with the token. Initial states are $t 0, t 1$. States are labeled with their active outputs. Edges are labeled with inputs, a missing input variable means "don't care". 'Burst4' means HBURST = BURST4, 'incr' means HBURST $=$ INCR, 'single' means neither of them. In the first step of decompositional synthesis states $t 0, . ., t 9$ were synthesized, in the second $t 10, . ., t 12$ were added, in the final step state $t 13$ was added.

where $\triangleright$ is ' $>$ ' if $b$ is an accepting state of $A_{\neg \varphi}$, else ' $\geq$ '. In words: for any state of the process template, and any transition of the automaton, if the current state of the product automaton is reachable, then the next state should also be reachable and the ranking function should be as stated.

The specification of AMBA we synthesize is derived from GR(1) specification. As a consequence it contain assumptions (A3, A6) of the form $\mathrm{G} \alpha(i)$ where $\alpha(i)$ is a Boolean formula over current inputs, and many guarantees $(\mathrm{G} 1, \mathrm{G} 4, \mathrm{G} 5, \mathrm{G} 6, \mathrm{G} 7, \mathrm{G} 8, \mathrm{G} 12, \mathrm{G} 10.2)$ of the form $\mathrm{G} \beta\left(i, o, o^{\prime}\right)$ where $\beta\left(i, o, o^{\prime}\right)$ is a Boolean formula over current inputs and outputs and next outputs. Instead of using the standard approach via automaton translation described above, we:

1. encode assertions of the form $\mathrm{G} \alpha(i)$ directly into SMT constraints, namely add $\alpha(i)$ to the the premise of the SMT rule. Thus, the premise becomes ' $\rho(a, q) \geq 0 \wedge o=\operatorname{out}(q) \wedge \alpha(\mathbf{i}) \rightarrow \ldots$ '

2. for all guarantees of the form $\mathrm{G} \beta\left(i, o, o^{\prime}\right)$ add SMT constraints of the form:

$$
\bigwedge q \in Q_{P} \bigwedge i \in \Sigma_{\mathrm{pr}}: \alpha(i) \rightarrow \beta\left(i, \text { out }(q), \text { out }\left(\delta_{P}(q, i)\right)\right)
$$

The first optimization is sound and complete, the second one introduces incompleteness.

For AMBA specification in Fig. 2 and 3 this optimization means that only guarantees G2, G3, G9, G10.1, G11 require the standard flow via automata translation.

Does this optimization help in the synthesis? Preliminary experiments (considering the first step of the decompositional synthesis of non-0 process) show:

- With the optimization the automaton for the negated specification has 24 states, without -42 states.

- The synthesis time with optimization is 16 minutes, without - 57 minutes. Interesting to note that the optimized and non-optimized versions spent the same time ( 2 minutes) checking satisfiability of the last query (with the model size of 10), so the main difference is in checking unsatisfiable 
Table 1: Results for non-0-process.

\begin{tabular}{c|cc} 
Additional assumptions & time & \#states \\
\hline $\begin{array}{c}\text { G HLOCK } \\
\text { G HBURST }=\text { BURST4 }\end{array}$ & $16 \mathrm{~min}$. & 10 \\
\hline G HBURST $=$ BURST4 & 13 sec. & 13 \\
\hline- (Full Specification) & 1 min. & 14
\end{tabular}

Table 2: Results for 0-process (bursts reduced: $3 / 4 \rightarrow 2 / 3$ ).

\begin{tabular}{c|cc} 
Additional assumptions & time & \#states \\
\hline $\begin{array}{c}\text { G HLOCK } \\
\text { GHBURST }=\text { BURST4 }\end{array}$ & $3 \mathrm{~h}$. & 11 \\
\hline G HBURST = BURST4 & $1 \mathrm{~min}$. & 11 \\
\hline- (Full Specification) & $1 \mathrm{~m} 30 \mathrm{~s}$. & 12
\end{tabular}

queries - Z3 identifies unsatisfiability of optimized queries faster (14 vs. 53 minutes). A similar behavior happens for a version of the same specification with reduced lengths of bursts $(3 / 4 \rightarrow$ 2/3): total times are $3 / 6$ minutes, but the last query took $1 \mathrm{~m} 40 \mathrm{~s} / 30$ s for optimized/non-optimized version.

Results. Synthesis times are in Tables 1 and 2 , the model synthesized for non-0-process is in Fig. 4 . The table has timings for the case when all optimizations described in this section are enabled - it was not our goal to evaluate the optimizations separately, but to find a combination that works for the AMBA case study.

For the 0-process we considered a simpler version with burst lengths reduced to $2 / 3$ instead of the original 3/4 ticks. With the original length the synthesizer could not find a model within 2 hours (it hanged checking 11 states models while the model has at least 12 states).

Without the decompositional approach, the synthesizer could not find a model for for non- 0 process of the AMBA specification within (at least) 5 hours.

\section{Conclusions}

We have shown that parameterized synthesis in token rings can be used to solve benchmark problems of significant size, in particular the well-known AMBA AHB specification that has been used as a synthesis benchmark for a long time. To achieve this goal, we extended slightly the cutoff results that parameterized synthesis is based on, and used a number of optimizations in the translation of the specification and the synthesis procedure itself to make the process feasible.

This is the first time that the AMBA case study, or any other realistic case study of significant size, has been solved by an automatic synthesis procedure for the general, parametric case. However, some of the steps in the procedure are manual or use an ad-hoc solution for the specific problem at hand, like the limited extension of cutoff results for global inputs, the construction of suitable functions to convert local to global outputs, or the decompositional synthesis for different grant schemes. Generalizing and automating these approaches is left for future work.

Furthermore, our synthesized implementation is such that the size of the parallel composition grows only linearly with the number of components. Thus, for this case study our approach does not only solve the problem of increasing synthesis time for a growing number of components, but also the problem of implementations that need an exponential amount of memory in the number of components. We pay for this small amount of memory with a less-than-optimal reaction time, as processes have to wait for the token in order to grant a request. This restriction could be remedied by extending the parameterized synthesis approach to different system models, e.g., processes that coordinate by guarded transitions, or communicate via broadcast messages.

Acknowledgments. We thank Sasha Rubin for insightful discussions on cutoff results in token rings. 


\section{References}

[1] Benjamin Aminof, Swen Jacobs, Ayrat Khalimov \& Sasha Rubin (2014): Parameterized Model Checking of Token-Passing Systems. In: VMCAI, LNCS 8318, Springer, pp. 262-281, doi 10.1007/978-3-642-540134_15.

[2] ARM Ltd. (1999): AMBA Specification (Rev.2). Available from www.arm.com.

[3] Tomás Babiak, Mojmír Kretínský, Vojtech Rehák \& Jan Strejcek (2012): LTL to Büchi Automata Translation: Fast and More Deterministic. In: TACAS, LNCS 7214, Springer, pp. 95-109, doi 10.1007/978-3-642-28756$5 \_8$.

[4] Roderick Bloem, Alessandro Cimatti, Karin Greimel, Georg Hofferek, Robert Könighofer, Marco Roveri, Viktor Schuppan \& Richard Seeber (2010): RATSY - A New Requirements Analysis Tool with Synthesis. In: CAV, LNCS 6174, Springer, pp. 425-429, doi:10.1007/978-3-642-14295-6_37.

[5] Roderick Bloem, Swen Jacobs \& Ayrat Khalimov (2014): Parameterized Synthesis Case Study: AMBA AHB (Extended Version). arXiv:1406.7608. Available at http://arxiv.org/abs/1406.7608.

[6] Roderick Bloem, Barbara Jobstmann, Nir Piterman, Amir Pnueli \& Yaniv Sa'ar (2012): Synthesis of Reactive(1) designs. J. Comput. Syst. Sci. 78(3), pp. 911-938, doi:10.1016/j.jcss.2011.08.007

[7] E. Allen Emerson \& Kedar S. Namjoshi (2003): On Reasoning About Rings. Int. J. Found. Comput. Sci. 14(4), pp. 527-550, doi:10.1142/S0129054103001881

[8] Bernd Finkbeiner \& Swen Jacobs (2012): Lazy Synthesis. In: VMCAI, LNCS 7148, Springer, pp. 219-234, doi:10.1007/978-3-642-27940-9_15.

[9] Bernd Finkbeiner \& Sven Schewe (2013): Bounded synthesis. STTT 15(5-6), pp. 519-539, doi $10.1007 / \mathrm{s} 10009-012-0228-\mathrm{z}$

[10] Yashdeep Godhal, Krishnendu Chatterjee \& Thomas A. Henzinger (2013): Synthesis of AMBA AHB from formal specification: a case study. STTT 15(5-6), pp. 585-601, doi:10.1007/s10009-011-0207-9

[11] Swen Jacobs \& Roderick Bloem (2014): Parameterized Synthesis. Logical Methods in Computer Science 10, pp. 1-29, doi: 10.2168/LMCS-10(1:12)2014.

[12] Barbara Jobstmann (2007): Applications and Optimizations for LTL Synthesis. Ph.D. thesis, Graz University of Technology.

[13] Ayrat Khalimov, Swen Jacobs \& Roderick Bloem (2013): PARTY Parameterized Synthesis of Token Rings. In: CAV, LNCS 8044, Springer, pp. 928-933, doi:10.1007/978-3-642-39799-8_66.

[14] Ayrat Khalimov, Swen Jacobs \& Roderick Bloem (2013): Towards Efficient Parameterized Synthesis. In: VMCAI, LNCS 7737, Springer, pp. 108-127, doi:10.1007/978-3-642-35873-9_9.

[15] Uri Klein \& Amir Pnueli (2010): Revisiting Synthesis of GR(1) Specifications. In: Haifa Verification Conference, LNCS 6504, Springer, pp. 161-181, doi:10.1007/978-3-642-19583-9_16.

[16] Leonardo de Moura \& Nikolaj Bjørner (2008): Z3: An Efficient SMT Solver. In: TACAS, LNCS 4963, Springer, pp. 337-340, doi $10.1007 / 978-3-540-78800-3 \_24$

[17] Amir Pnueli \& Roni Rosner (1990): Distributed Reactive Systems Are Hard to Synthesize. In: FOCS, IEEE Computer Society, pp. 746-757, doi $10.1109 /$ FSCS.1990.89597 